\title{
Hardware Design of Wireless Physical Token Ring System for Mobile and Cell Communication
}

\author{
${ }^{1}$ Nidal F. Shilbayeh and ${ }^{2}$ Mahmoud Z. Iskandarani \\ ${ }^{1}$ Applied Science University, Department of Computer Science, \\ P.O. Box 41, Post Code: 11931, Amman, Jordan \\ ${ }^{2}$ Alzaytoonah University of Jordan, \\ Faculty of Science and Information Technology, \\ P.O. Box: 911597, Post Code: 11191, Amman-Jordan
}

\begin{abstract}
A wireless network system based on an infrared and Laser scanning Physical Token Ring architecture (W-PTRS) was designed, built and tested successfully. The network provides a different way of utilizing the concept of a token ring network but with a higher efficiency related to the speed of data transfer and the ability to parallel process client-server requests leading to a new concept of the token ring system, which will not fail simply because of ring breakage. The study proposes a new wireless communication protocol, which is neither IEEE802.11 nor Bluetooth. This newly developed protocol could prove to be an excellent communication media if supported by industry. If implemented, this wireless system will provide an alternative but efficient way of wireless communication.
\end{abstract}

Key words: Cellular Network, Mobile Communication, Token Ring, Wireless LAN, Protocols, Infrared

\section{INTRODUCTION}

In the last few years a new type of Local Area Network (LAN) has appeared ${ }^{[5,6]}$. This new type of LAN, which is the wireless LAN, provided an alternative to the traditional LANs based twisted pair, coaxial cable and optical fiber. The wireless LAN serves the same purpose as that of a wired LAN and that of conveying information among devices attached to the LAN. But with the lack of physical cabling to tie down the location of a node on a network, the network can be much more flexible whereby moving a wireless node is easy, as opposed to the large amount of labor required to add or move the cabling in any other type of network. Also going wireless may be a better choice where the physical makeup of the building makes it difficult or impossible to run wire in the building.

Wireless is ideal for portable computers, using wireless connections allow portable computers to still be portable without sacrificing the advantages of being connected to a network. Wireless can also be used in combinations with cabled LAN. In that all the machines that will require relative mobility will be connected wirelessly. Wireless communications use transmission techniques such as Spread Spectrum, Narrow Band Microwave, Infrared and Laser ${ }^{[16,17]}$.

In this study a new approach to wireless communication networking is carried out. This approach is based on using infrared and laser devices to carry out network communications whereby the behavior of the designed system emulates the token ring protocol, as the transmission will not begin until the server(s) accept a token binding from a client(s), which is achieved using an infrared and laser locking system in the network.

A wireless Local Area Network (LAN) is a flexible data communications system implemented as an extension to or as an alternative for, a wired LAN. Using advanced communication technology, wireless LANs transmit and receive data over the air, minimizing the need for wired connections. Thus, wireless LANs combine data connectivity with user mobility.

Wireless LANs have gained strong popularity in a number of vertical markets, including the healthcare, retail, manufacturing, warehousing and academia. These industries have profited from the productivity gains of using handheld terminals, notebook computers and cellular mobile phone to transmit real time information to centralized hosts for processing. Today wireless LANs are becoming more widely recognized as a general-purpose connectivity alternative for a board range of applications.

The widespread reliance on networking in business and meteoric growth of the internet and online services are strong testimonies to the benefits of shared data and shared resources. With wireless LANs, users can access shared information without looking for a place to plug in and network managers can set up or augment networks without installing or moving wires. Wireless LANs offer mobility, installation speed, simplicity, installation flexibility and reduced cost-of-ownership.

Overview of wireless LAN technology: Manufacturers of wireless LANs have a range of technologies to choose from when designing a wireless LAN solution. 
Each technology comes with its own set of advantages and limitations ${ }^{[8-22]}$.

Narrow band technology: A narrow band radio system submits and receives user information on a specific radio frequency. Narrow band radio keeps the radio signal frequency as narrow as possible just to pass the information. Undesirable cross talk between communications channels is avoided by carefully coordinating different users on different channel frequencies.

Spread spectrum technology: Most wireless LAN systems use spread-spectrum technology; a wideband radio frequency technique developed by the military for use in reliable, secure, mission-critical communications systems. Spread-spectrum is designed to trade off bandwidth efficiency for reliability, integrity and security. In other words, more bandwidth is consumed than in the case of narrowband transmission, but the tradeoff produces a signal that is, in effect, louder and thus easier to detect, provided that the receiver is not tuned to the right frequency, a spread-spectrum signal looks like background noise. There are two types of spread spectrum radio: frequency hopping and direct sequence.

Frequency-hopping spread spectrum technology: Frequency-Hopping Spread-Spectrum (FHSS) uses a narrowband carrier that changes frequency in a pattern known to both transmitter and receiver. Properly synchronized, the net effect is to maintain a single logical channel. To an unintended receiver, FHSS appears to be short-duration impulse noise.

Laser communication system: Laser Technology is cost effective option for short-haul connectivity. The technology received ill-deserved comments regarding performance. However, when properly installed and maintained its very reliable and efficient.

The key item for determining whether laser technology is appropriate for an application is path distance. Since this technology is visual, it is imperative that clear line of sight is achieved and maintained. An additional consideration is the stability of the mounting surface/structure. Any building sway, normally associated with tall structures, must be avoided or considered in the installation and equipment choice. Furthermore, the systems may be compared to a light pipe-what is actually input is received at the other end of the path.

In addition to orientation and mounting conditions, a major cause of ineffective performance is attempting to extend the technology beyond its distance limitations.

Despite the design considerations mentioned, a well planned installation can provide bandwidths up to $155 \mathrm{Mbps}$ and beyond.
Narrow band microwave: Microwave technology is not really a LAN technology. Its main use is to interconnect LANs between buildings. This requires microwave dishes on both ends of the link. The dishes must be in line-ofsight to transmit and collect the microwave signals. Microwave is used to bypass the telephone company when connecting LANs between buildings.

One major drawback to the use of microwave technology is that the frequency band used requires licensing by the FCC. Once a license is granted for a particular location, that frequency band cannot be licensed to anyone else, for any purpose, within a 17.5 mile radius.

Infrared: Infrared LANs use infrared signals to transmit data. This is the same technology used in products like remote controls for television and VCRs. These LANs can be setup using either a point-to-point configuration or a sun-and-moon configuration where the signals are diffused by reflecting them off of some type of surface.

The major advantage of infrared is its ability to carry a high bandwidth, but its major disadvantage is that they can easily be obstructed, since light cannot pass through solid objects.

System design: In a typical wireless LAN configuration, a transmitter/receiver (transceiver) device, called an access point connects to the wired network from a fixed location using standard cabling, at a minimum, the access point receives, buffers and transmits data between the wireless LAN and the wired network infrastructure. A single access point can support a small group of users and can function within a range of less than one hundred to several hundred feet. The access point (or the antenna attached to the access point) is usually mounted high but may be mounted essentially anywhere that is practical as long as the desired radio coverage is obtained ${ }^{[1-4]}$.

End users access the wireless LAN through wireless- LAN adapters, which are implemented as PC cards in a notebook or palmtop computer, as cards in desktop computers, integrated within handheld computers, or integrated with cellular phones. Wireless LAN adapters provide an interface between the client Network Operating System (NOS) and the airwaves via an antenna. The nature of the wireless connection is transparent to the NOS.

Hardware design: The system consists of laser diode based transceivers connected to both clients and servers. The server transceiver is mounted on a circular plate with a software controlled DC-Motor used to move it in a circular motion covering 360 degrees. In effect, emulating a token ring system. Table 1 summarizes the system components. 
Table 1: System components

\begin{tabular}{|c|c|c|}
\hline Component Name & Numbers & Description \\
\hline $\begin{array}{l}\text { Binary Ripple } \\
\text { Counter }\end{array}$ & 74HC/HCT4040 & $\begin{array}{l}12 \text { stage binary ripple } \\
\text { counters with a clock } \\
\text { Input, an overriding } \\
\text { Asynchronous master } \\
\text { Reset input and twelve } \\
\text { Parallel outputs }\end{array}$ \\
\hline $\begin{array}{l}\text { Dual D-type } \\
\text { Flip-flop }\end{array}$ & HEF4013 & $\begin{array}{l}\text { Dual D-type flip-flop } \\
\text { which features independent } \\
\text { set direct, Clear direct, } \\
\text { clock inputs and outputs }\end{array}$ \\
\hline AND & HEF4081B & $\begin{array}{l}\text { Positive quadruple } 2 \\
\text {-input AND function }\end{array}$ \\
\hline Binary Counter & 4060 & $\begin{array}{l}\text { Binary counter with } \\
\text { three oscillator terminals } \\
\text { (RS, RTC and CTC), ten } \\
\text { buffered outputs (O3-O9 } \\
\text { and O11 to O13) and an } \\
\text { overriding asynchronous } \\
\text { master reset input }\end{array}$ \\
\hline Multiplexer & 74HC/HCT151 & $\begin{array}{l}8 \text { input high speed Si-gate } \\
\text { CMOS devices }\end{array}$ \\
\hline Shift register & 74HC/HCT595 & $\begin{array}{l}\text { 8-Bit serial/serial or } \\
\text { parallel-out shift register } \\
\text { with output } \\
\text { latches; Tri-state }\end{array}$ \\
\hline Hex Inverter & MCI4069UB & Hex Inverter \\
\hline $\begin{array}{l}\text { Operational } \\
\text { Amplifiers }\end{array}$ & TL074 & $\begin{array}{l}\text { Low noise operational } \\
\text { amplifier with low } \\
\text { input BIOS and Offset } \\
\text { currents and fast slew rate }\end{array}$ \\
\hline Voltage Regulators & LM7805 & Three terminal regulators \\
\hline $\begin{array}{l}\text { Eight Darlington } \\
\text { Arrays }\end{array}$ & UTC2803 & $\begin{array}{l}\text { High-voltage, high-current } \\
\text { Darlington Arrays drivers } \\
\text { comprised of } 8 \text { NPN } \\
\text { Darlington pairs }\end{array}$ \\
\hline $\begin{array}{l}\text { Stepping motor } \\
\text { driver }\end{array}$ & BA6846FS & $\begin{array}{l}\text { Motor driver with a } \\
\text { maximum output current } \\
\text { of } 0.5 \mathrm{~A} \text { incorporating } \\
\text { special logic which } \\
\text { allows } 3 \text { modes of } \\
\text { operations: forward, } \\
\text { reverse and power save }\end{array}$ \\
\hline
\end{tabular}

Software design: Firstly, the software waits for the client to switch a light $\mathrm{ON}$ to begin transmission, after that the server reads the file name which must be transmitted and then the client sends a signal to the server that it is ready for transmission and waits a replying signal. After that the server sends a zero signal to indicate the start-bit, followed by seven-bits indicating the stop bits.

The client sends a signal to indicate that it received the data and then it switches off and start the scanning process (Token Passing) again.

The data are transmitted bit by bit using parallel port by shifting the data bit by bit seven times. Hence, the system makes use of the functionality of the parallel port to connect multi client-server system that transmits the wireless data serially. Figure 1 and 2 represent the transceiver protocol ${ }^{[7-10]}$.

The transceiver of the designed system is based on generating and receiving signals that are compatible with the parallel port. A sensor receives the signal; the output of the sensor is buffered via D-Flip-Flops and directed to the parallel port status register to be read by the software.

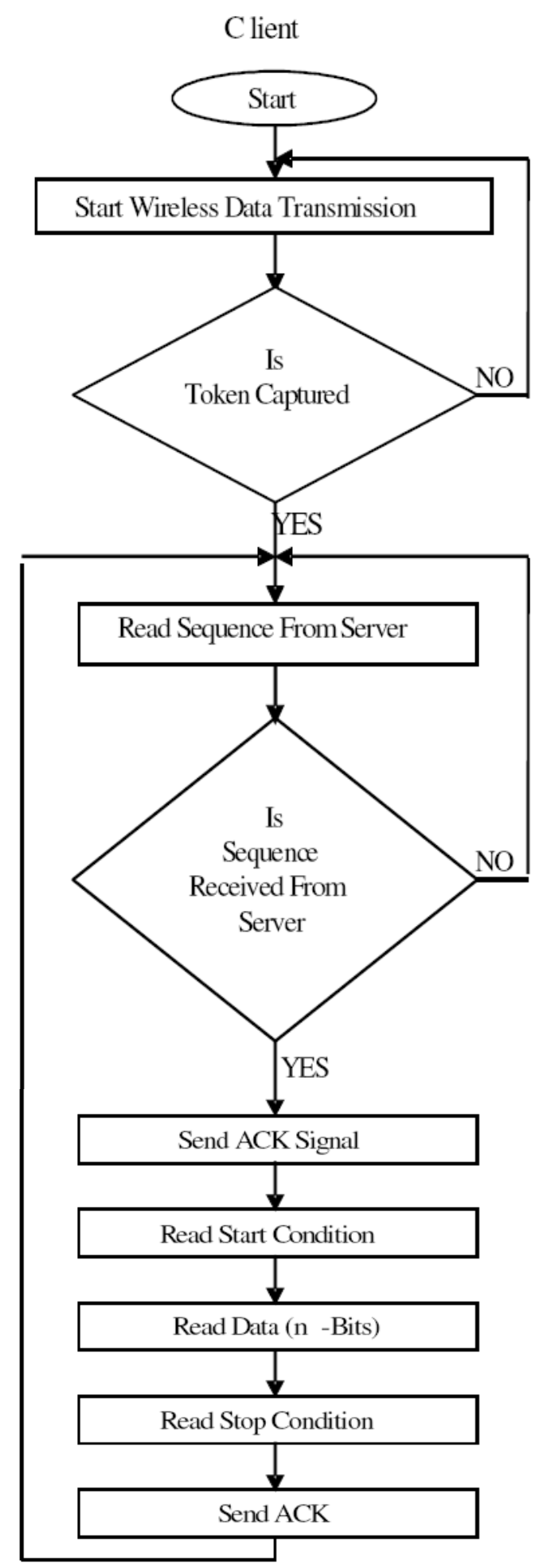

Fig. 1: The client transceiver algorithm

In transmitting the data from the parallel port output, a signal driver consists of D-Flip-Flop that provides the required signal for the transmitting diode. A Voltage regulator is used to provide the ICs and diode with stable $5 \mathrm{~V}$ voltage source. 


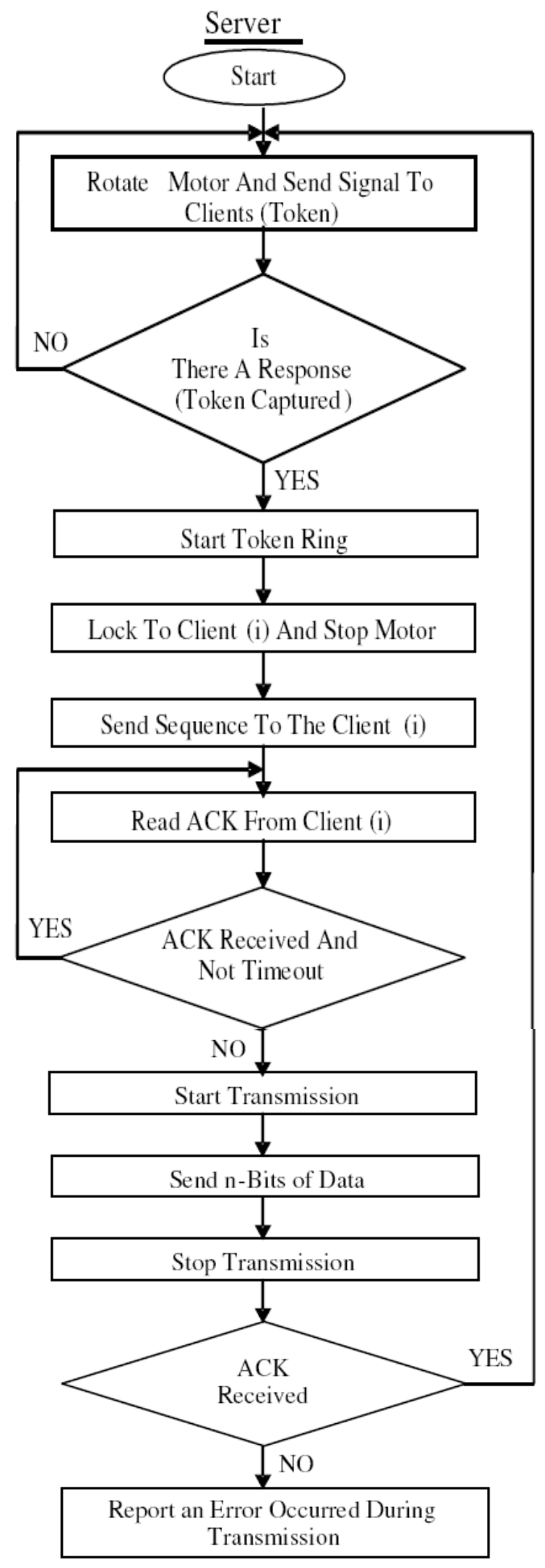

Fig.2: Client transceiver system

Locked system is used to control the starting of the transceiver, which consists of stepper motor and a light sensor. The client consists of wireless signal transceiver circuit with a light source; the client's light is switched ON when the user wants to communicate with the server. The stepper motor rotates with a sensor light to seek for clients. The motor stop rotating when it finds a light, it stops the rotation and sends a signal to the client and waits for a hand shaking signal from the client, when the hand shaking signal is received, a transceiver protocol is started and begin files transmission. After ending the transmission, the light of a client is switched OFF and the motor begins to rotate seeking other clients.

The server controls and rotates the scanning system in search for clients requesting data communication. Once it finds the clients it stops the rotation and locks in preparation for data exchange to start. Figure 5 shows the interfacing module that synchronizes and controls the transmission and reception process.

The circuit employs the 74HC/HCT151, 8-inputs multiplexer to implement a parallel to serial signal conversion (data selection) and interface control signals generated via D-Flip-Flops with clock signal generated from the 4060 binary counters. For the communication process to take place between the server and the clients, synchronization has first to occur as follows:

- A pulse from the computer to clear previous signal resets the handshaking Flip-Flop

- A waiting period is assigned to the output of the handshaking Flip-Flop to change from 0 to 1 for transmission process to start

- Once the Flip-Flop output switches from 0 to 1 , the computer will output data and resets the hand shaking flip-flop signal again

- The process continues until a complete frame is transmitted

The data is synchronized and buffered by the Dflip flop devices (HEF4013B) as shown in Fig. 5. Before being selected by the multiplexer, the data format allows 25\% for low, 25\% for high logic levels, $50 \%$ for data. The data signal is converted to optical form for wireless transmission to take place as shown in Fig. 7. The transmitted signal is modulated to $38 \mathrm{KHz}$ in order to avoid interference from surrounding devices. At the receiving end the computer reconstructs the transmitted frame and extracts the data.

The receiving computer awaits the start of the bit stream by monitoring the output of the bit synchronization process of D-Flip-Flop via the input/output port. When the output goes high, it signals the start of bits arrival, the received data signals are sampled in the middle to ensure stability, the receiving PC the resets the hand shaking line waiting for new inputs. Changing the status of the D-flip-flop from 0-1 signals the end of the frame. 
American J. Appl. Sci., 1 (3), 176-183, 2004

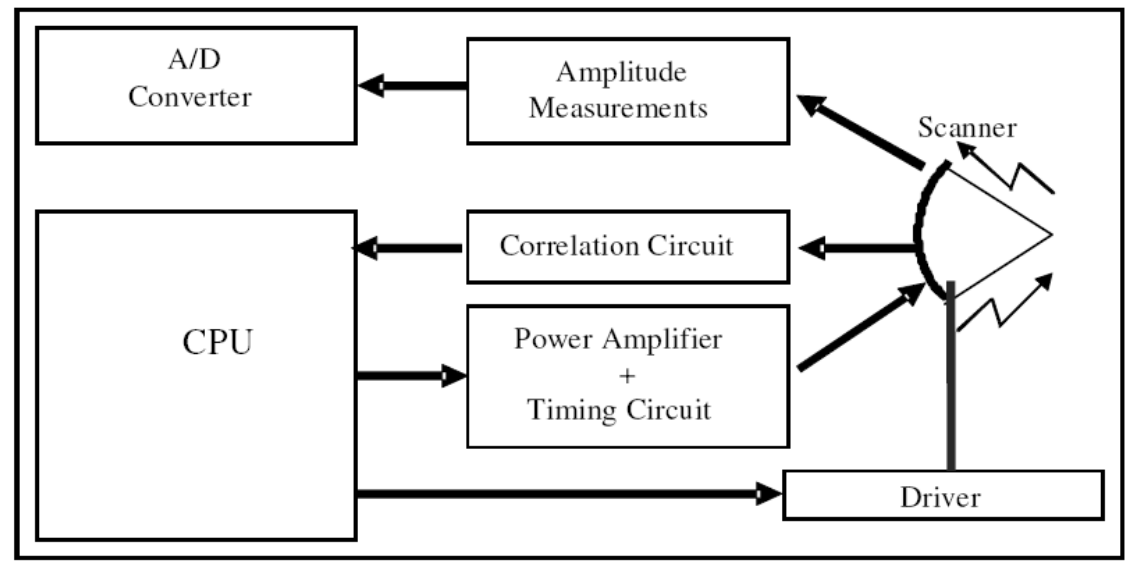

Fig. 3: Server transceiver and scanner control system

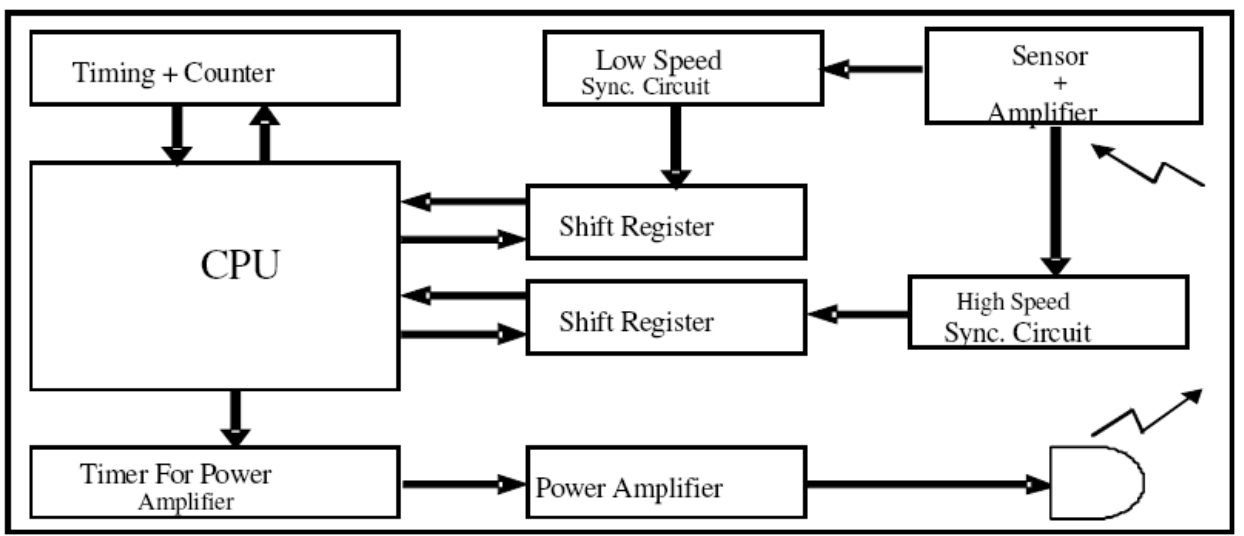

Fig. 4: Client transceiver system

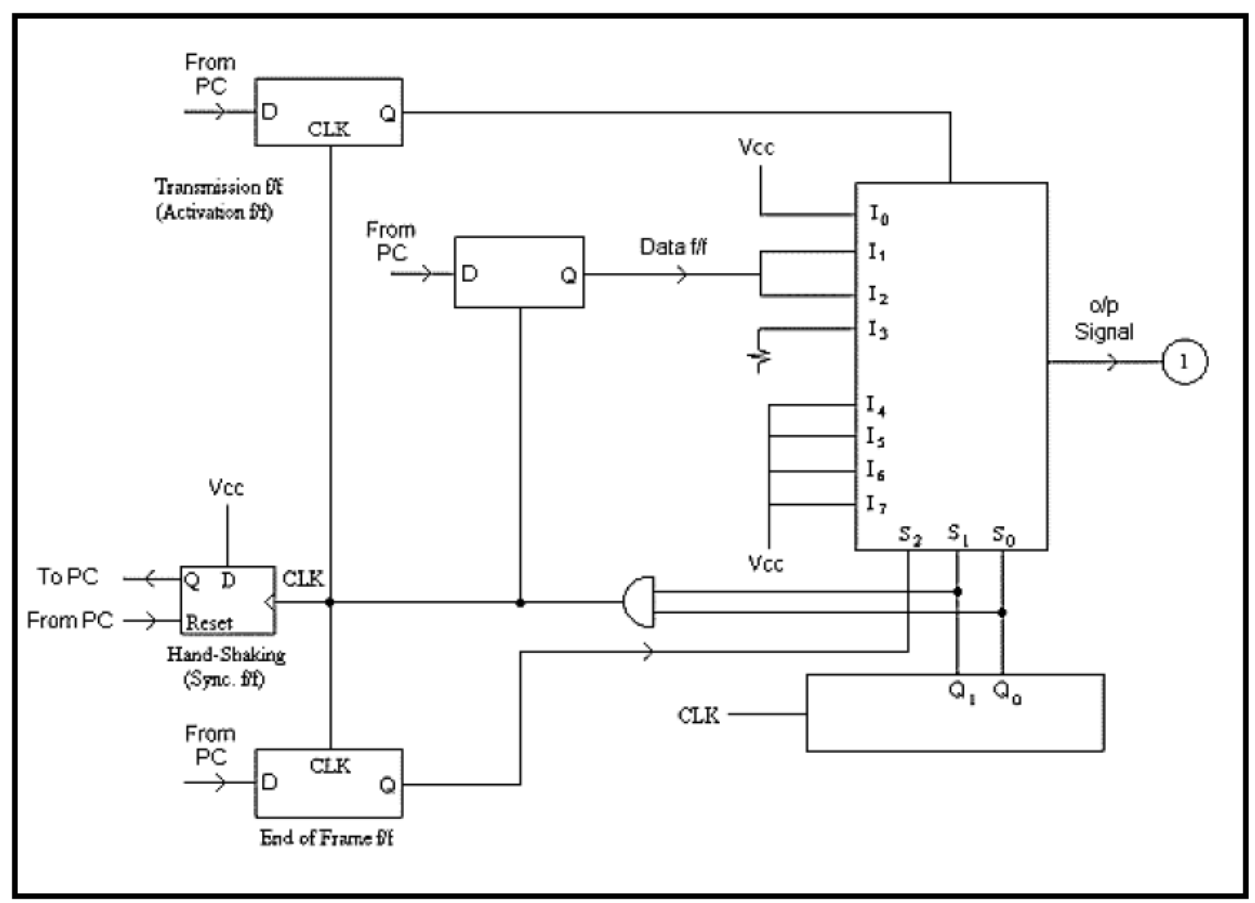

Fig. 5: Interfacing and synchronization system 


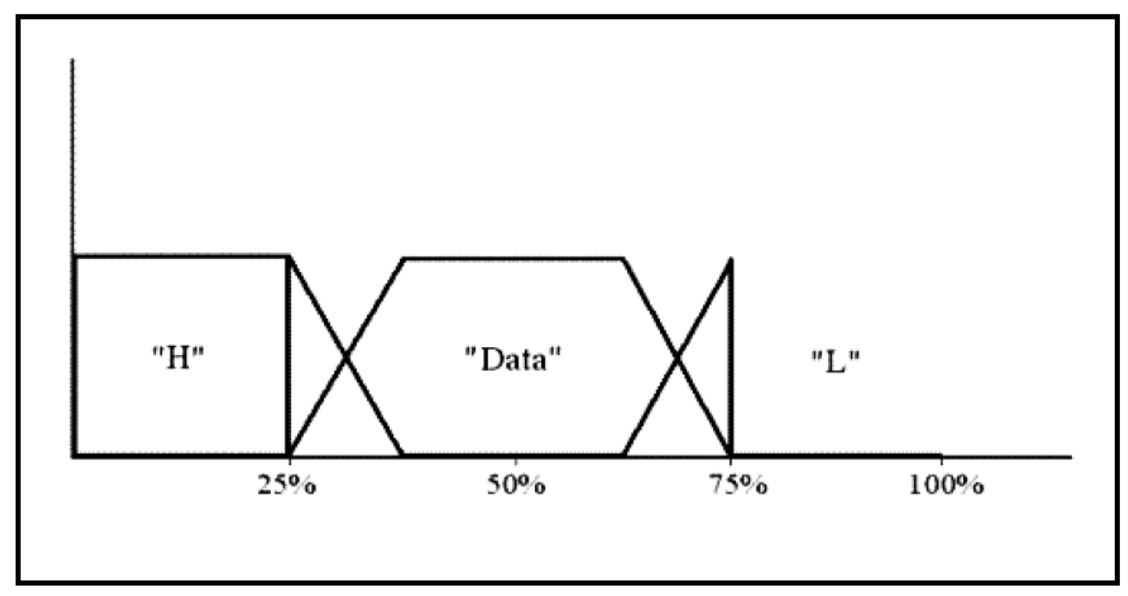

Fig. 6: Transmitted data format

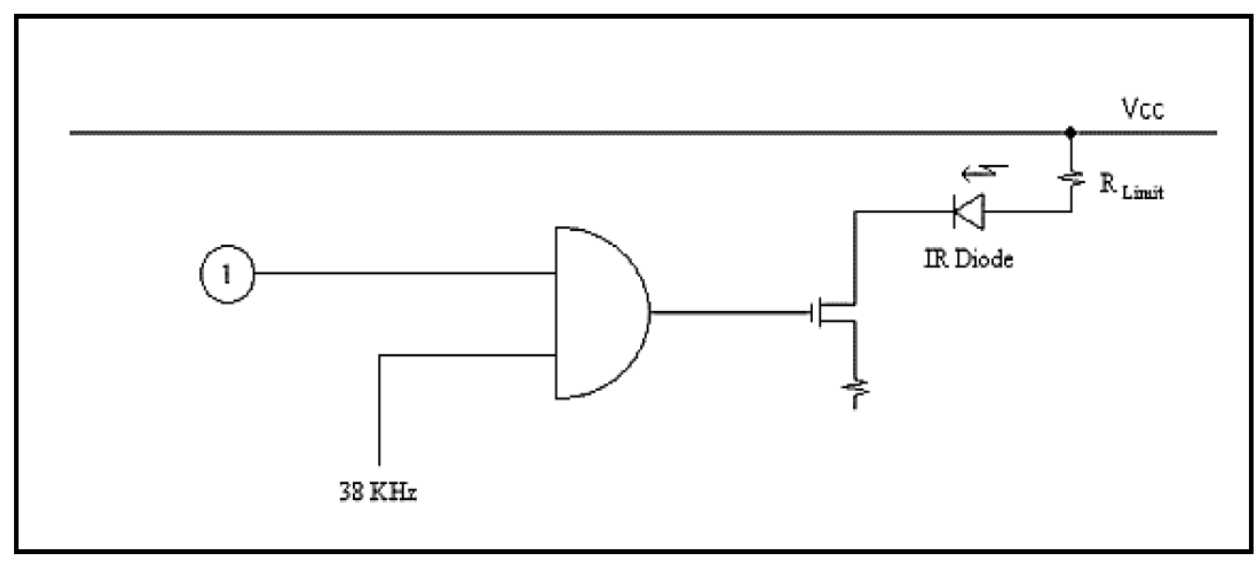

Fig. 7: Optical transmission circuit

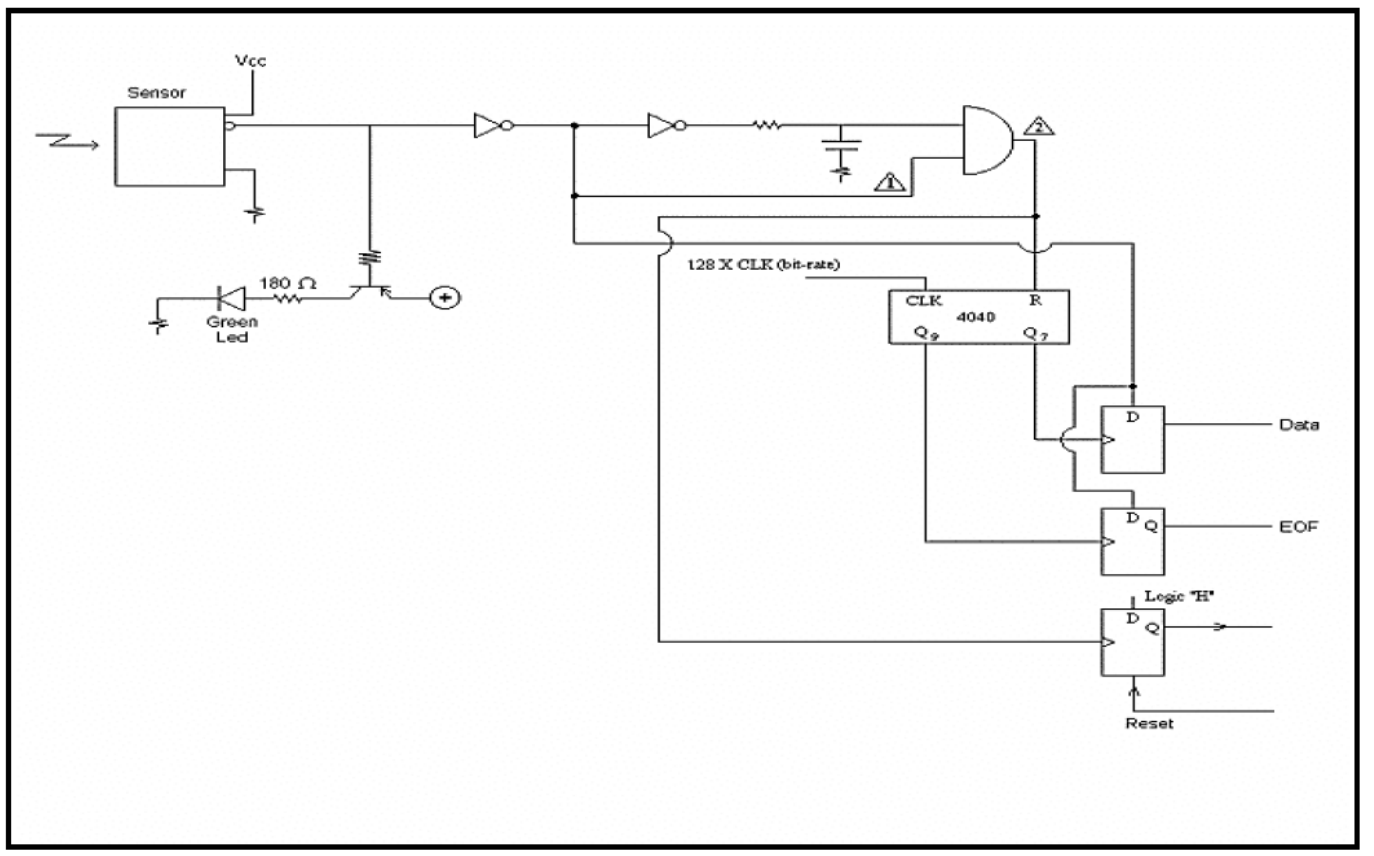

Fig. 8: Optical receiving circuit 


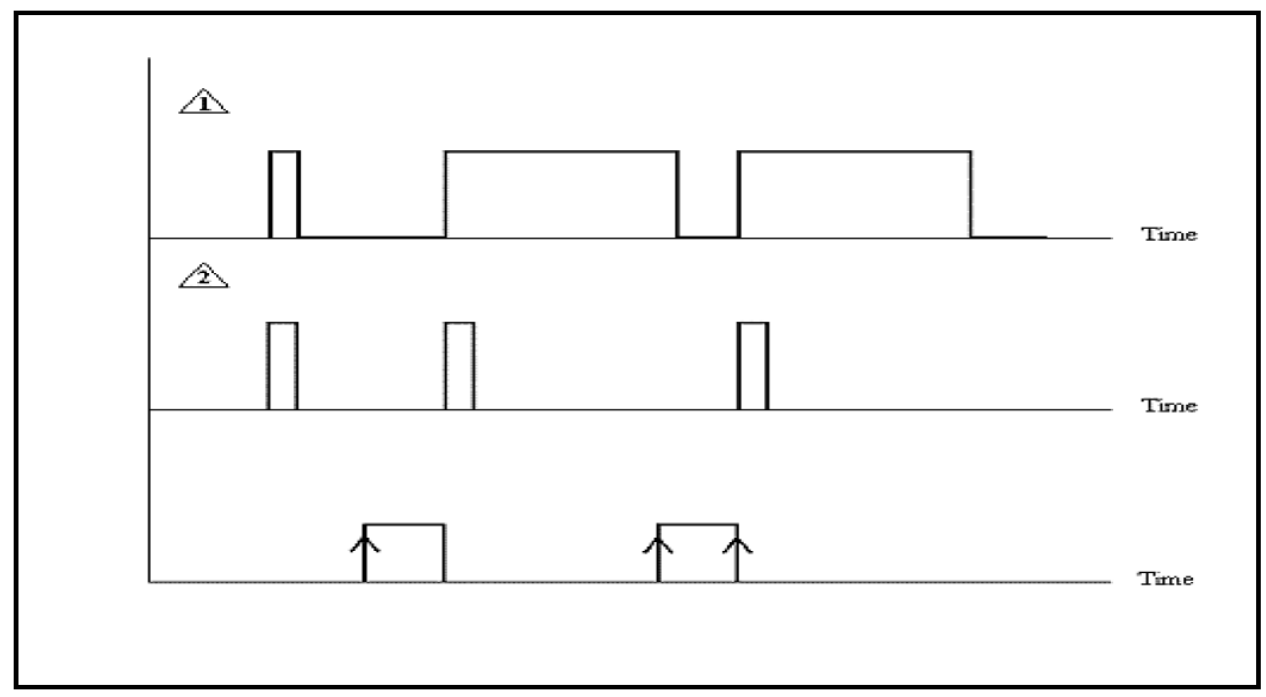

Fig. 9: Received data format

\section{CONCLUSION}

The designed W-PTRS, which was successfully built and tested, proved to be an excellent tool as a nonstandard wireless LAN application. In addition, it serves as a connectivity network for anyone with a device equipped with an IR TXIRX system that can connect to it and access network and internet services. This is much like "a stop station" or transit station provides parallel Client-Server connectivity, which can be modeled as a multiple ring arrangement. The concept of Multi-Token Rings (MTR) is a very important topic to research into, in terms of wireless efficiency and data security, especially when more than one type of wireless sensors is used as in our system. As a new protocol, the system can provide a very efficient way of data communication between clients and servers. It does however need to be supported and standardized in order for people to feel its real benefits.

\section{REFERENCES}

1. IEEE, 1999. Standard for Wireless LAN Medium Access Control (MAC) and Physical Layer (PHY) specifications, ISO/IEC 8802-11.

2. Karp, B. and H. T. Kung, 2000. GPSR: Greedy Perimeter Stateless Routing for Wireless Networks, In Proc. ACM/IEEE, MobiCom.

3. Crow, B.P. and J.G. Kim, 1997. IEEE 802.11 Wireless Local Area Networks, IEEE Communications Magazine.

4. Nee, R. and G. Awater, 1999. New High-Rate Wireless LAN Standards, IEEE Communications Magazine, pp. 116-126.

5. Anastasi, G. and L. Lenzini, 2000. QoS Provided by IEEE 802.11 , Wireless LAN to Advanced Data Applications: A Simulation Analysis. ACM Wireless Networks 6: 99-108.
6. Gupta, P. and P. R. Kumar, 2000. The Capacity of Wireless Networks, IEEE Transactions on Information Theory, 46: 388-404.

7. Ho, T. and K. Chen, 1996. Performance Analysis of IEEE 802.11; CSMA/CA Medium Access Control Protocol. Proc. PIMRC'1996. Taipei, Taiwan, pp. 407-411.

8. Bianchi, G., L. Frattaand and M.Oliveri, 1996. Performance Evaluation and Enhancement of the CSMA/CA MAC Protocol for 802.11 Wireless LANs. Proc. PIMRC'1996. Taipei, Taiwan, pp. 392-396.

9. Huang, K. and K.Chen, 1995. Interference Analysis of Nonpersistent CSMA with Hidden Terminals in Multicell Wireless Data Networks, Proc. PIMRC 1995, Toronto, 13: 907-911, Environments. IEEE JSAC.

10. Gerla, M., R. Bagrodia, L. Zhang, K. Tan and L. Wang, 1999. TCP over wireless Multi-hop Protocols: Simulation and Experiments, ICC'99, pp.1089-1094.

11. Xylomenos, G. and G. C. Polyzos, 1999. TCP and UDP Performance over a Wireless LAN, Proc. IEEE, INFOCOM'99.

12. $\mathrm{Xu}, \mathrm{S}$. and T. Saadawi, 2001. Does the IEEE 802.11 MAC Protocol Work Well in Multihop Wireless Ad hoc Network. IEEE Communications Magazine, 39 (6).

13. Jain, R. 1991. The Art of Computer Systems Performance Analysis, John Wiley and Sons.

14. Xylomenos, G. and G. C. Polyzos, 1999. Link Layer Support for Quality of Service on Wireless Internet Links, IEEE Personal Communications, 6: 52-60.

15. Barakat, C., E. Altman and W. Dabbous, 2000. On TCP Performance in a Heterogeneous Network: A Survey. IEEE Communications Magazine, pp. 4046. 
16. Pahlavan, K. and A. Levesque, 1994. Wireless data communications, Proceedings of the IEEE 82:1398-1430.

17. Pahlavan, K., T. Probert, and M. Chase, 1995. Trends in Local Wireless Networks, IEEE Communications Magazine, 33: 99-108.

18. Jabbari, B. 1995. Network Issues for Wireless Communications, IEEE Communications Magazine, 33: 88-98

19. Peter T. Davis and Craig R. McGriffin, 1994. Wireless Local Area Networks: Technology, Issues \& Stratigies, McGraw-Hill.
20. Bud Bates, 1994. Wireless Networked Communications Concepts, Technology \& Implementation, McGraw-Hill.

21. Akyildiz, I. F., W. Su, Y. Sankarasubramaniam, and E. Cayirci, 2002. Wireless Sensor Networks; A Survey, Computer Networks, 38 (9).

22. Manzoni B. and G. Cano, 2003. Providing interoperability between IEEE 802.11 and Blue Tooth Protocols For Home Area Networks, Computer networks, 42: 23-37. 